\title{
Numerical calculation of zeros and weights for Gaussian quadrature: Legendre polynomials
}

\section{H. A. Harutyunian, H. A. Poghosyan}

NAS RA V.Ambartsumian Byurakan Astrophysical Observatory (BAO), Armenia E-mail: hhayk@bao.sci.am

\begin{abstract}
The roots of the Legendre polynomials and corresponding weights necessary for Gaussian quadrature of integrals are calculated numerically. A package of program created for high accuracy calculations is used for the machine computing. The numerical procedure used for determination of the Legendre polynomials' roots and corresponding weights is described. We also bring here some results of numerical calculations as an illustration.
\end{abstract}

Key words: Gaussian quadrature: roots of Legendre polynomials - high accuracy calculations: long numbers.

\section{Introduction}

Machine computing is always approximate, and the accuracy of calculations decreases with the number of mathematical acts conducted during numerical procedures. There are two main sources of inaccuracy affecting the results. One is coming from the mathematical algorithms transforming the analytical formulae into constructed artificially discrete schemes of step-by-step approaching. The next one results from the machine representation of numbers with limited quantity of significant digits. Researchers apply a variety of methods to diminish the calculation errors of both types.

Errors of the second type are most often encountered in theoretical calculations, when dealing with numerical values that differ by many orders of magnitude. If one is sure that neglecting the smallest terms in such expressions does not affect the result, no problem arises. However, it often happens that the numerical result is in order comparable to the smallest members of the expression. Then such neglect is unacceptable, and one needs to represent the main members of the expression with a larger number of significant figures.

A few decades ago, one of the authors (H.A.H) has developed a package of program, which allows one computing with arbitrary long (with many significant digits) numbers. Thanks to the mentioned package, some machine computation 
algorithms are realized, which would be impossible with ordinary or double precession regimes of modern computers. Particularly, zeros of Hermite polynomials with the corresponding weights are calculated for up to $120^{\text {th }}$ order (see Poghosyan 2017; Harutyunian, Poghosyan 2018).

Here we present the results of calculations of zeros and weights of Legendre polynomials. Both the zeros of Hermite polynomials and the zeros of Legendre polynomials are calculated for usage in the computational procedures of numerical integration when solving the Ambartsumian's functional equations. The zeros of Hermite polynomials and linked weights are used for the case of frequency redistribution, while second set is used for discretization of angular integrals.

For the purpose mentioned above, we use the package of long number computational mathematics giving opportunity for calculations with many (in the given version - up to 200) significant digits.

\section{Legendre polynomials}

Legendre polynomials representing the polynomial solution of Legendre equation allow the following presentation by Rodrigues formula(see, for example, Abramowitz, Stegun 1964):

$$
P_{n}(x)=\frac{1}{2^{n} n !} \frac{d^{n}}{d x^{n}}\left(x^{2}-1\right)^{n} .
$$

It is well known that the Legendre polynomials constitute a complete and orthogonal system. Their orthogonality can be expressed as follows:

$$
\int_{-1}^{1} P_{m}(x) P_{n}(x) d x=\frac{2}{2 n+1} \delta_{m n},
$$

where $\delta_{m n}$ is the Kronecker symbolequal to 1 if $m=n$ and to 0 otherwise.

One can immediately check that the Legendre polynomials obey the following recurrence relation:

$$
(n+1) P_{n+1}(x)=(2 n+1) x P_{n}(x)-P_{n-1}(x),
$$

which is easily applicable to numerical calculations if the expressions for the first polynomials

$$
\begin{aligned}
& P_{0}(x)=1 ， \\
& P_{1}(x)=x
\end{aligned}
$$

are used for the beginning steps.

The roots of a Legendre polynomial can be calculated using the Newton universal iterative method 


$$
x_{i}^{(k+1)}=x_{i}^{(k)}-\frac{P_{n}\left(x_{i}^{(k)}\right)}{P_{n}^{\prime}\left(x_{i}^{(k)}\right)},
$$

where the indices $i$ and $(k)$ show the root number and the number of the next iteration. The derivative $P_{n}^{\prime}(x)$ is determined from relation

$$
P_{n}^{\prime}(x)=\frac{n}{1-x^{2}}\left[P_{n-1}(x)-x P_{n}(x)\right] .
$$

To implement the numerical procedure according to (6) - (7), as a zero iteration, the numerical value of the following expression is usually calculated:

$$
x_{i}^{(0)}=\cos \left[\pi \frac{4 i-1}{4 n+1}\right] \text {. }
$$

However, one can use another procedure for computing the roots of polynomials. That is the direct method, which we applied for finding the roots of Hermie polynomials. For all polynomials, we used the same procedure, starting calculation of the given polynomial for the argument $x=0$. In the next step, we increment the argument by some small value $\Delta x$ and again determine the value of the polynomial. The increment $\Delta x$ must be small enough not to jump over the two consecutive roots of a polynomial at once. Then, if the sign of the calculated value is the same with the previous one, the next step is identical. If sign changes, we put $\Delta x \rightarrow-\frac{\Delta x}{2}$ and continue procedure in the same way. The iteration process considered complete if the increment becomes smaller than the required accuracy for the roots.

This mathematical algorithm gives rather good results. However, one needs to compare the speeds of convergence for two methods to choose the best one. Actually, we did not put such a purpose. Our purpose was calculation anyhow of the zeros and corresponding weights using the "long numbers" mathematics.

\section{Numerical results}

All calculations have been carried out using the package HAHMATH (see Poghosyan 2017, Harutyunian, Poghosyan 2018) with long numbers given by at least 116 significant digits. We followed the iterative method described in the previous section. An iterative process is considered complete if, in the next iteration step, the increment of the argument in order of magnitude is equal to or less than the required accuracy.

We present here only positive roots of polynomials, since

$$
P_{n}(-x)=(-1)^{n} P_{n}(x) \text {. }
$$


Table 1. Zeros and weights of the 49 and 50 order Legendre polynomials

0. 6.34206849826867860288348208784849731-2 $1.26585997269672051067985288575536190-1$ $1.89241592461813586485310173859986511-1$ 2.51135178612577273507155942824611274-1 3.12017532119748762207860676506416265-1 3.71643501262284888863734540946960527-1 4.29772993341576524658584141807423033-1 4.86171941452492042176976096058575062-1 5.406132469917260665582254672294355916-1 $5.92877694108900712455864336792628534-1$ 6.42754832419237664056856948583900109-1 6.90043824425132113504751807274941927-1 7.34554254237402696213674212858524884-1 $7.76106894345446635018142812248456071-1$ 8.145344273598554315395007877647777044-1 8.49682119844165701034881872242979333-1 8.81408445573008910037031535817004926-1 $9.09585655828073285213019640355800071-1$ $9.34100294755810149058982460636544902-1$ 9.54853658674137233555243679668184419-1 9.71762200901555380139972421504774133-1 $9.84757895914213004359298995368574710-1$ 9.93788661944167790760113859257953618-1 $9.98820150606635379361831272704110655-1$ $3.10983383271888761123289896659491942-2$ 9.31747015600861408544503776396003478-2 $1.54890589998145902071628620941109501-1$ 2.16007236876041756847284532617101333-1 2.76288193779531990327645278521130185-1 3.35500245419437356836988257291071697-1 3.93414311897565127394229253823817270-1 4.498063349740387891471314677783758817-1 $5.04458144907464201651459131849141192-1$ $5.57158304514650054315522909625801607-1$ 6.07702927184950239180381796391832893-1 6.55896465685439360781624864003679819-1 7.01552468706822251089546257883655728-1 $7.44494302226068538260536252682194242-1$ 7.84555832900399263905305196340991200-1 $8.21582070859335948356254110873939537-1$ $8.55429769429946084611362643934757467-1$ 8.85967979523613048637540982466753634-1 9.13078556655791893089735642771657094-1 9.36656618944877933780874947272496602-1 9.566109552428079429977456441566222094-1 9.72864385106692073713344104606252053-1 $9.85354084048005882309009625632489404-1$ 9.94031969432090712585108200420694728-1 $39.98866404420071050185459444974218505-1$
3.17316407023952988591267339397648174-2 $6.33355092964917485908369274000905201-2$ 6.29527074651956994743995557828910063-2 $6.23164173200572674010768252159497042-2$ $6.14292009791929362968266461403010749-2$ 6.02946309531520173031061168755415904-2 $5.89172757600272660245276507424925162-2$ $5.73026815301874754851645923508165363-2$ $5.54573496748035886904315814826980672-2$ $5.33887107082589685279429337301762061-2$ 5.11050943301445906746228060493185613-2 4.86156958878282402776511972878685308-2 4.59305393555958535424996199721276710-2 4.30604369812595979883454840010384958-2 4.00169457663730213686050367431033214-2 3.68123209630006898194672366988822950-2 3.34594667916221743424871508916892018-2 2.99718846205838253506905580741472100-2 2.63636189270660169609457452397420476-2 2.26492015874466764987709642160428873-2 1.88435958530894584444506533911073492-2 1.49621449356246510295843191202015040-2 1.10205510315935804975082881668333327-2 7.03509959008645147345067831134841086-3 3.02727898892290507748069817582714097-3 6.21766166553472623210331073606134308-2 6.19360674206832433840875097808306885-2 $6.14558995903166637564067860839153750-2$ 6.07379708417702160317500153848110016-2 $5.97850587042654575095764053125852307-2$ $5.86008498132224458351224366308484662-2$ 5.71899256477283837230293150659931630-2 5.55577448062125176235674256122694975-2 5.37106218889962465234587972556645527-2 5.16557030695811384899052958400952796-2 4.94009384494663149212435807514327286-2 4.69550513039484329656330136349876825-2 4.43275043388032754920222868303941974-2 4.15284630901476974224119789640670178-2 3.85687566125876752447701502363859348-2 3.54598356151461541607346110009757970-2 3.22137282235780166481658273230039534-2 2.88429935805351980299063731132324325-2 2.53606735700123904401948783854427234-2 2.17802431701247929815920690626903412-2 1.81155607134893903512599434223546198-2 $1.43808227614855744193789089273243499-2$ $1.05905483836509692635696814992410223-2$ 6.75979919574540150277887817798503180 2.90862255315514095840072434285548080 
One can check the accuracy of numerical results using, for example, relations

$$
\int_{0}^{1} P_{0}(x) d x=1
$$

and

$$
\int_{0}^{1} P_{1}(x) d x=\frac{1}{2}
$$

One needs to replace integrals (11)-(12) by their Gaussian quadrature, and then carry out the following required computations:

$$
\operatorname{err}(1)=1-\sum_{k=1}^{n} w_{k}
$$

and

$$
\operatorname{err}(2)=\frac{1}{2}-\sum_{k=1}^{n} x_{k} w_{k},
$$

where $n$ is the number of nodes $x_{k}$, and the quantities $w_{k}$ are the corresponding weights.

It is worth noting on the base of our numerical computations that the accuracy of (12) is much lower comparing with one found for (13). Havingnodes of polynomials and the corresponding weights determined using numbers with eighty significant digits and accurate to the thirtieth digit, the quadrature (12) provides accuracy up to $10^{-3}$ only and almost does not depend on $n$ in the range $10 \leq n \leq 25$ .Equally does not depend on $n$ also the accuracy of (13). However, in the last case the accuracy reaches to $10^{-30}$.

\section{Conclusion}

The program package HAHMATH created for calculations with long numbers is a rather useful tool for numerical computations when a large number of significant digits are required for providing the needed accuracy of results. We used it for calculating the nodes of the Legendre polynomials and corresponding weights. Having these quantities one can examine numerically the accuracy of Gaussian quadrature for various dependences of integrands on the argument.

The iterative method used here for numerical procedures gives acceptable results. Nonetheless, one needs to compare it with the Newton iterative method for choosing the most effective one. Authors intend to make such a comparison in one of forthcoming papers. 


\section{References}

Abramowitz, M., Stegun, I. A., 1964, Handbook of Mathematical Functions with Formulas, Graphs and Mathematical Tables, Washington Harutyunian, H.A., Poghosyan, H.A., 2018, Astrofizika, 2018 (in press)

Poghosyan, H.A., 2017, ComBAO, LXIV, 41 